\title{
Nanobeacon: A time calibration device for KM3NeT
}

\author{
Diego Real ${ }^{1,}$, David Calvo ${ }^{1}$ on behalf of the KM3NeT collaboration \\ ${ }^{1}$ Instituto de Física Corpuscular de Valencia, Catedrático José Beltrán, 2, 46980 Paterna, Spain
}

\begin{abstract}
A very large volume neutrino telescope is being constructed in the Mediterranean Sea by the KM3NeT collaboration. Thousands of glass spheres holding a set of 31 small-area photomultipliers will be deployed at high depth forming a three-dimensional matrix. The glass spheres are the basic elements that will detect the Cherenkov light induced by charged particles produced in neutrino interactions with the surrounding matter. Time synchronization between photomultipliers is required to achieve optimal performance. Due to the high volume to be covered by KM3NeT, a cost reduction of the different systems is a priority. To this end a very low price calibration device, the so called Nanobeacon, has been designed and developed. In this article the main characteristics of the device are described.
\end{abstract}

\section{Introduction}

The KM3NeT telescope [1] is a deep sea neutrino detection infrastructure being deployed in the Mediterranean Sea. The operation of the telescope is based in the interaction of the neutrino with the material surrounding the detectors that can produce a muon, which travelling through the detector with a higher speed than the light speed in water induces Cherenkov radiation, a weak blue light which propagates through the detector illuminating it. This radiation is detected by the Photomultipliers (PMT) housed in the main element of the telescope, the DOM (Digital Optical Module) [2]. The DOMs register the arrival time of the Cherenkov light, allowing the reconstruction of the muon trajectory and, therefore, the neutrino's trajectory. The angular resolution of the reconstructed track depends on the accurate measurement of the arrival time of Cherenkov photons reaching the PMTs. Good time and position calibration of the detector is therefore of utmost importance to achieve a good angular resolution.

Water-based neutrino telescopes have intrinsic and unavoidable limitations in the time precision like, for example, the chromatic dispersion and scattering of light in water $(\sigma \sim 2$ ns for a travelling distance of $50 \mathrm{~m}$ ), and the combined effect of the PMT transit time spread and electronics response $(\sigma \sim 1.5 \mathrm{~ns})$. Taking into account these intrinsic limitations, the required precision for a time calibration system to measure the relative time between photo-sensors should be $\sigma \leq 1 \mathrm{~ns}$. Light Emission Diode (LED) located within the DOM produce short duration and powerful light pulses which are detected by the PMTs allowing

*Corresponding author: real@ific.uv.es 
the measurement of the time delay between the arrival of the photon to the photocathode and the time stamping in the front-end electronics. LED beacons will be used, in the so called Nanobeacon, to calibrate Digital Optical Modules in the same Detection Unit of the KM3NeT.

The main aim of the Nanobeacon is to perform the calibration between the optical elements of the same line. The Nanobeacon contains a LED pointing upwards; when the appropriate command is received, the LED emits an ultra-short light pulse. The Nanobeacon has appeared as the logical evolution of the LED Beacon used in ANTARES [3]. The mechanical integration inside the DOM avoids the need of using a mechanical container for the device which reduces substantially the cost. Moreover, thanks to new types of LEDs and to the opto-mechanical coupling of the LED the configuration selected for the Nanobeacons increases more than 50 times the light yield of the ANTARES LED Beacon.

\section{Nanobeacon}

The Nanobeacon pulser is an evolution of the Kapustinsky circuit which has been modified for KM3NeT. The trigger of the pulse is generated by the Central Logic Board (CLB). It provides a square pulse of configurable width mixed to the direct current voltage provided by the power supply rail. The direct current voltage loads a capacitor that is discharged through the LED during the switch on/off of two transistors which work in opposition. The switching of the transistors is triggered by a signal provided by the FPGA, causing the discharge of the capacitor through the LED and the emission of a narrow optical pulse. The trigger generated by the FPGA is provided to the Nanobeacon pulser by means of a three wire twisted cable in order to reduce the electromagnetic induced noise. This connection also provides ground and the power supply. In the figure 1 the Nanobeacon board is shown.

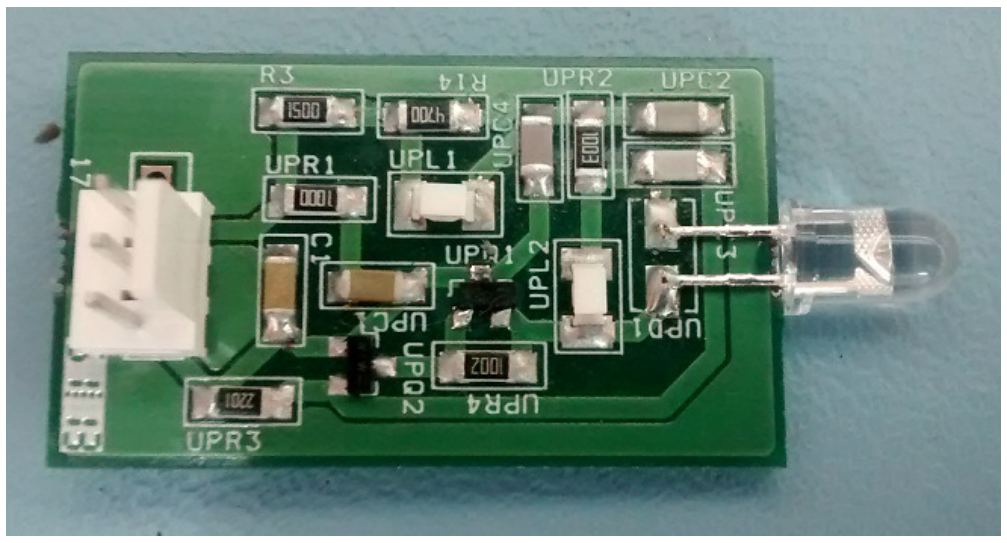

Figure 1. The Nanobeacon pulser board for the KM3NeT

\section{The LED}

Several LED models have been tested in the laboratory. According to the studies carried out in terms of amplitude and rise time of the emitted pulses and angular distribution of light, a HLMP-CB1A-XY0DD was selected to be used in the Nanobeacon device [4]. Comparing with the original LEDs used in ANTARES, these new models are more powerful but have a smaller opening angle. The main attributes of the LED are shown in Table 1. 
Table 1. Main characteristics of the HLMP-CB1A-XY0DD LED used in the Nanobeacon

\begin{tabular}{|c|c|}
\hline Attribute & Value \\
\hline Color & Blue \\
\hline Wavelength $(\mathrm{nm})$ & 470 \\
\hline Viewing angle $\left({ }^{\circ}\right)$ & 15 \\
\hline Luminity intensity $(\mathrm{mcd})$ & 12000 \\
\hline Package & $\mathrm{T}-13 / 4(5 \mathrm{~mm})$ \\
\hline
\end{tabular}

\section{Firmware}

An Intellectual Property (IP) has been implemented in the CLB to generate the trigger signal needed by the Nanobeacon. This core is connected with a wishbone bus, where five registers of 32 bits are accessible to configure the Nanobeacon trigger signal. Specifically, the first register allows to enable and to disable the trigger using one of its bits, the so called Enable control bit. With another bit, Enable power, it is possible to disable the power supply of the Nanobeacon. There are, therefore, two different ways to disable the Nanobeacon. A second register allows to define the delay to start flashing after the time slice. In a third register, it is defined the Nanobeacon pulse width The configuration of the flashing frequency in defined in a fourth register and the number of triggers per time slice in a fifth one. Table 2 describes the different registers that configure the IP core that controls the Nanobeacon and in figure 2 it is exemplified the behavior of the Nanobeacon firmware.

Table 2. Description of the Nanobeacon control registers

\begin{tabular}{|c|c|c|}
\hline Reg. & Name & Remark \\
\hline 0x00 & Enable register & Bit 0: Enable - Bit 1: Power - Bits (31-2): Don't care \\
\hline 0x01 & Delay after Time Slice & In 16 ns ticks (default 0 ns) \\
\hline 0x02 & Nanobeacon pulse width & In 16 ns ticks (default 64 ns) \\
\hline 0x03 & Nanobeacon pulse frequency & In 16 ns ticks (default 20 kHz) \\
\hline 0x04 & Number of pulses & Number of pulses (default 100 pulses) \\
\hline
\end{tabular}




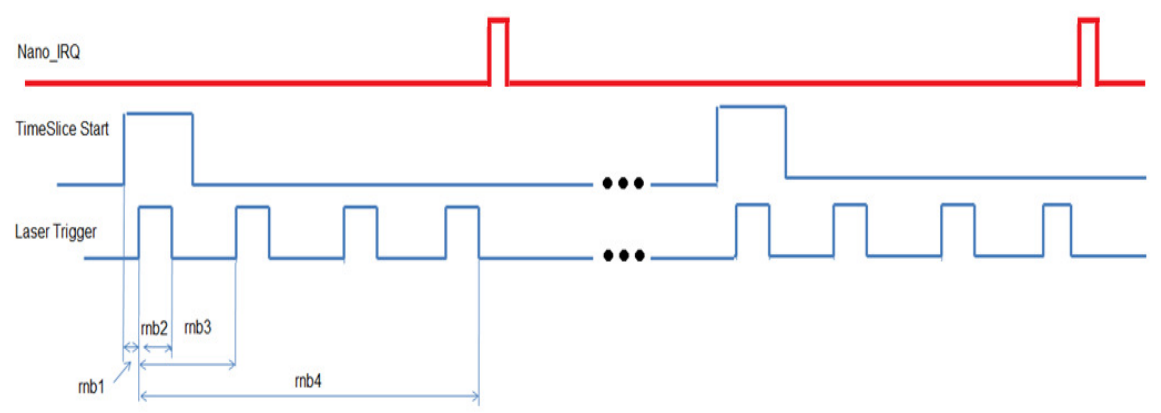

Figure 2. Example of the behaviour of the Nanobeacon firmware with respect to the time slice.

\section{Conclusions}

One of the most important devices for time calibration in KM3NeT has been presented. The basic electronics together with the firmware used for the operation of the board has been described. A new powerful model of LED has been chosen for the KM3NeT neutrino telescope. An IP core has been implemented in the firmware of the CLB to fully control the behaviour of the emitted pulse of light within the time slice. Future works will imply the evaluation of the energy emission in the pulse shape and the evaluation in terms of wavelength, rise time and FWHM of LED with different wavelength.

\section{Acknowledgement}

The authors acknowledge the financial support of the funding agencies: Agence Nationale de la Recherche (contract ANR-15-CE31-0020), Centre National de la Recherche Scientifique (CNRS), Commission Europèenne (FEDER fund and Marie Curie Program), Institut Universitaire de France (IUF), France; 'Helmholtz Alliance for Astroparticle Physics', Germany; Istituto Nazionale di Fisica Nucleare (INFN), Ministero dell'Istruzione, dell'Università e della Ricerca (MIUR), Italy; Agence de l'Oriental and CNRST, Morocco; Stichting voor Fundamenteel Onderzoek der Materie (FOM), Nederlandse organisatie voor Wetenschappelijk Onderzoek (NWO), the Netherlands; Plan Estatal de Investigación (refs. FPA2015-65150-C3-1-P,-2-P and -3-P, (MINECO/FEDER)), Severo Ochoa Centre of Excellence and MultiDark Consolider (MINECO), and Prometeo and Grisolía programs (Generalitat Valenciana), Spain.

\section{References}

1. S. Adrian-Martinez et al. [KM3NeT collaboration], Letter of Intent for KM3NeT 2.0, Journal of Phys. C 43373 (2016)

2. M. Circella, P. Coyle, P. Kooijman, The Digital Optical Module (DOM) for the KM3NeT Detector, 33rd ICRC, Rio de Janeiro (2013)

3. J.A. Aguilar et al. [ANTARES collaboration], Time calibration of the ANTARES neutrino telescope, Astroparticle Physics 34, 539-549 (2011)

4. D. Real, D. Calvo [KM3NeT collaboration], The time calibration system of the KM3NeT: The Laser Beacon and the Nanobeacon, Ad-hoc Network and Wireless. ADHOC-NOW (2014) 